\title{
In situ electrode manipulation for three terminal field emission characterization of individual carbon nanotubes
}

\author{
R. C. Smith, ${ }^{\text {a) }}$ J. D. Carey, D. C. Cox, and S. R. P. Silva \\ Nano-Electronics Centre, Advanced Technology Institute, School of Electronics and Physical Sciences, \\ University of Surrey, Guildford GU2 7XH, United Kingdom
}

(Received 22 November 2005; accepted 2 July 2006; published online 8 August 2006)

\begin{abstract}
In situ three terminal electron field emission characterization of an isolated multiwalled carbon nanotube has been performed, where both anode and gate electrodes are attached to high precision piezodrivers. All measurements are performed in a scanning electron microscope allowing accurate knowledge of the local environment of the nanotube to be obtained. It is shown that the presence of the grounded gate electrode screens the applied field by approximately $32 \%$. This technique in positioning the gate and anode electrodes allows for an estimate of the gate transparency factor and demonstrates characterization of individual carbon nanotubes without the need for fabrication of arrays of emitters. (C) 2006 American Institute of Physics. [DOI: 10.1063/1.2335604]
\end{abstract}

Since the identification of carbon nanotubes (CNTs), ${ }^{1}$ there has been sustained research into numerous potential applications of CNTs, in particular, for applications in field emission. ${ }^{2,3}$ The high aspect ratio of a CNT results in a high local electric field for a given macroscopic field. As a result their use as electron sources for field emission based flat panel displays has been greatly explored, with the emergence of prototype displays. ${ }^{4}$ While extensive two terminal field emission measurements have been performed, ${ }^{2-6}$ considerably fewer three terminal studies ${ }^{7-10}$ have been undertaken and those that have been performed have often been from arrays of nanotubes. In this letter we demonstrate a method of examining the gated field emission properties of an individual CNT and a method for establishing the gate transparency factor and the shielding of the emitter due to the presence of the gate itself. By performing the measurements in a scanning electron microscope (SEM) with electrodes attached to manipulators, we are able to investigate individual emitters, with a degree of freedom to move the electrodes in real time. Our approach opens the possibility of an effective method for testing the performance of a range of emitter structures prior to fabricating a prototype device, where the characteristics of an ensemble of emitters are measured. In addition, an ability to test single emitters may lead the way to enhance the emission uniformity from CNTs and increase the fraction of nanotubes that are actively emitting.

Multiwalled carbon nanotubes were synthesized by a plasma arc discharge system between two graphite electrodes. The subsequent carbon deposit was purified by microfiltering and oxidization at $500^{\circ} \mathrm{C}$ to remove any amorphous carbon and carbon particulates, leaving purified CNTs. The CNTs were mixed into a polymer solution of polystyrene, which was dissolved in toluene. An ultrasonic treatment was used to improve the dispersion of the CNTs within the polymer. Vacuum casting methods were then used and the as-cast films were hot pressed to remove any residual solvent. To expose a single CNT the sample was mechanically broken and the broken edge studied in a Cambridge Instruments Stereoscan 250 III scanning electron microscope. A single CNT with a height of $1.5 \mu \mathrm{m}$ and a radius of approximately $40 \mathrm{~nm}$ was found. This emitter was chosen as

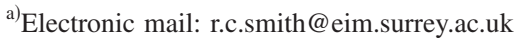

it was perpendicular in orientation to the substrate. The surrounding area around the emitter was carefully scanned approximately $100 \mu \mathrm{m}$ in all directions to ensure that there were no CNTs present in all directions from the chosen emitter. This is an important step as neighboring CNTs could affect the emission results by field screening, ${ }^{6}$ and that the presence of any other CNTs beneath the gate electrode would complicate our results.

Two piezoelectric micromanipulators were mounted inside the SEM chamber to control the position of both the anode and gate electrodes with an accuracy of $40 \mathrm{~nm}$ in all directions. ${ }^{11}$ The anode electrode used was a sharpened tungsten tip with end radius of $5 \mu \mathrm{m}$. Further details of the experimental setup are described elsewhere. ${ }^{11}$ The gate electrode used was manufactured in a FEI Nova 600 dual beam focused ion beam (FIB) system to create a freestanding metal structure with a $1 \mu \mathrm{m}$ hole. A tungsten tip with end radius of $5 \mu \mathrm{m}$ was physically bent at the tip and its sidewalls etched by $30 \mathrm{keV} \mathrm{Ga}$ ions to create a $1.5 \mu \mathrm{m}$ thin metal film. The electrode was then rotated through $90^{\circ}$ and a $1 \mu \mathrm{m}$ hole etched through by a similar process. The resulting structure can be seen in the inset to Fig. 1(a), where the view is down the inside of the probe so that the $1 \mu \mathrm{m}$ hole can be clearly observed. The gate and anode electrodes were mounted on the micromanipulators within the SEM and positioned 1 and $40 \mu \mathrm{m}$ above the CNT tip, respectively, as shown in Fig. 1(a). It is important to note that the large $(30 \mu \mathrm{m})$ structure to the center left of Fig. 1(a) was examined for any CNTs (not shown here), which could possibly interfere with emission testing. However, no CNTs were observed in this area and we conclude that this feature does not affect our emission results. Figure 1(b) shows a higher magnification SEM image of the gate electrode above the CNT. The gate to CNT separation used was $1 \mu \mathrm{m}$ and the threshold voltage is defined for $10 \mathrm{nA}$ of emission current.

Field emission characterization was performed on the setup of Fig. 1(a) using two computer controlled Keithley 238 high current source meters connected to the substrate and gate, and a Keithley 237 high voltage source meter connected to the anode. The SEM beam current was turned down during testing as to not interfere with emission. Initially, the substrate and gate were held at $0 \mathrm{~V}$ and the anode voltage ramped from 0 to $500 \mathrm{~V}$, in $25 \mathrm{~V}$ steps. A current 

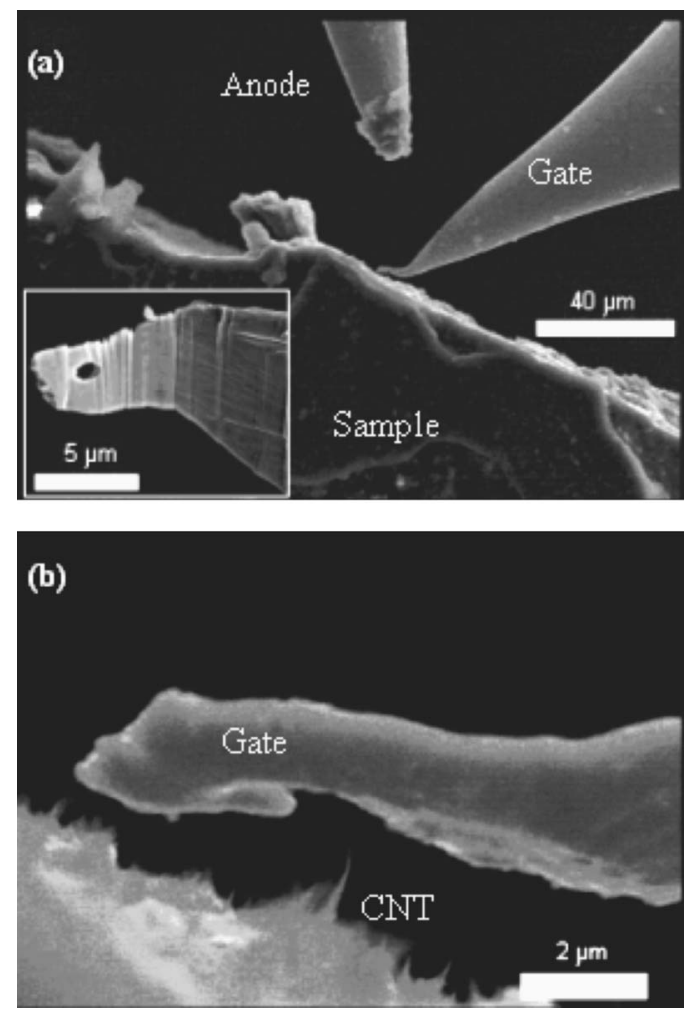

FIG. 1. (a) Scanning electron microscope image of the experimental setup. Anode electrode is seen at the top of the image, $40 \mu \mathrm{m}$ from the CNT. The gate electrode is positioned $1 \mu \mathrm{m}$ above the CNT tip. The inset shows a view down the inside of the gate electrode and shows the $1 \mu \mathrm{m}$ hole clearly. (b) SEM image of the gate electrode positioned $1 \mu \mathrm{m}$ above the CNT emitter.

limit of $1 \mu \mathrm{A}$ was employed to reduce the risk of sample damage. The variation of the anode current with anode voltage can be seen in Fig. 2(a). At $0 \mathrm{~V}$ gate bias $\left(V_{G}\right)$, an emission current of $10 \mathrm{nA}$ is reached at an anode voltage, $V_{A}$, of $340 \mathrm{~V}$ and for a $1 \mu \mathrm{A}$ emission current, an anode voltage of $460 \mathrm{~V}$ is required. The gate bias was then increased from 5 to $25 \mathrm{~V}$ in $5 \mathrm{~V}$ steps, with measurements of the anode current as a function of anode voltage made at each gate voltage. It is apparent from Fig. 2(a) that the maximum current is reached at steadily decreasing anode voltages from 460 to $180 \mathrm{~V}$ as the gate voltage is increased. The emission characteristics of Fig. 2(a) were subjected to a FowlerNordheim analysis with resultant plots shown in Fig. 2(b). Assuming a work function of $5 \mathrm{eV}$, enhancement factors of $620,1749,1773,1696,2049$, and 1920 are found for gate voltages of $0,5,10,15,20$, and $25 \mathrm{~V}$, respectively. The apparent enhancement factor of 620 calculated at $0 \mathrm{~V}$ gate bias assumes that the whole of the anode field (voltage) is dropped across the vacuum gap and neglects the effects of field screening due to the presence of the metal gate suspended above it. For this reason $I$ - $V$ measurements were performed with the gate electrode removed, as shown in Fig. 2(a) (data marked with a star). For a current of $10 \mathrm{nA}$, a voltage of $230 \mathrm{~V}$ is required, rising to $313 \mathrm{~V}$ for the maximum current of $1 \mu \mathrm{A}$. It is also important to note that the removal of the gate electrode does not increase the threshold field. If the results seen so far were due to emission from the gate electrode, rather than the CNT, then one might expect a large increase in threshold field by removing the gate. As this is clearly not seen here, we are confident that the electron source is the CNT, not the gate. Performing a FowlerNordheim analysis on this data reveals an enhancement Downloaded 30 Mar 2009 to 131.227.178.132. Redistribution subject
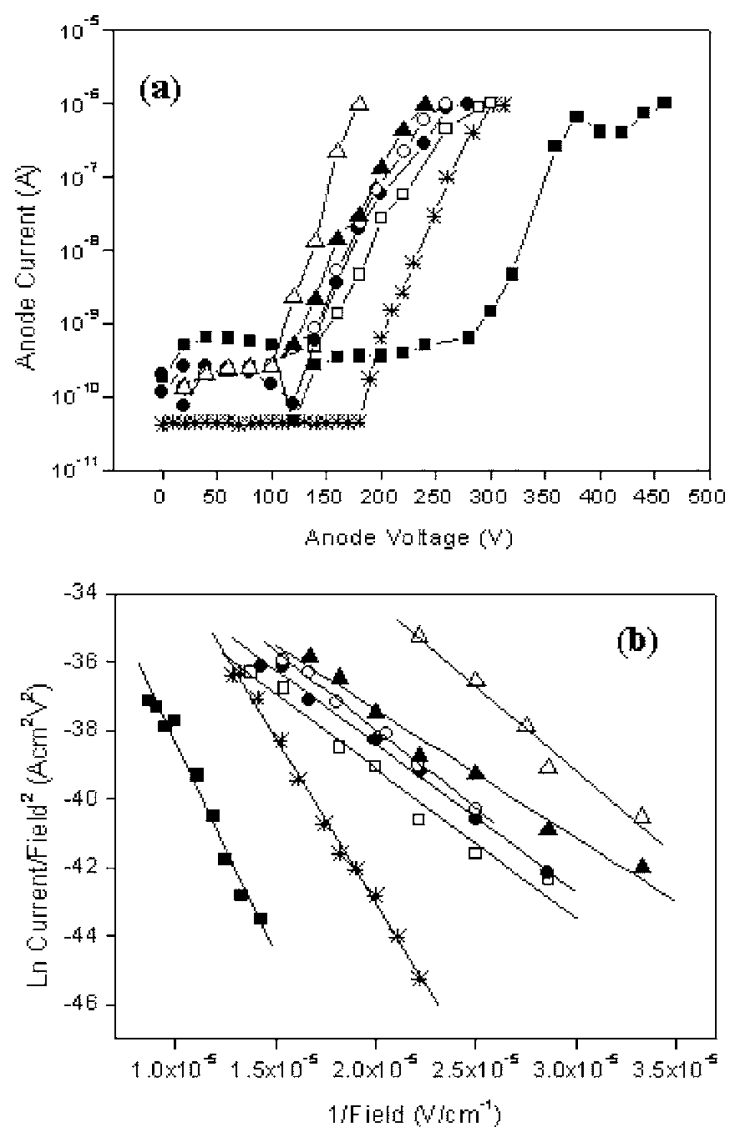

FIG. 2. (a) Anode current against anode voltage for gate biases of 0 (घ), 5 $(\square), 10(\bullet), 15(\bigcirc \mathrm{tm}), 20(\mathbf{\Delta})$, and $25 \mathrm{~V}(\triangle)$. Emission with gate removed is also shown $(*)$. (b) Fowler-Nordheim analysis of the data of (a).

factor of 794. This value of enhancement factor is intrinsic to the nanotube under investigation (depending on the length and radius). Armed with a knowledge of the applied voltage, $V_{a}$, and the enhancement factor, it is possible to calculate the local threshold field, $E_{L}=\beta V_{a} / D$, where $D$ is the interelectrode spacing $(40 \mu \mathrm{m})$. For the case of two terminal measurements, the local field for a $10 \mathrm{nA}$ current is $4.57 \mathrm{~V} / \mathrm{nm}$. This is the field that will be required to extract the same current for the unbiased three terminal structure. In such a situation, it is possible to introduce a screening factor due to the gate electrode $(1-\eta)$, such that,

$$
E_{a} \beta(1-\eta)=E_{L}
$$

where $E_{a}$ is the value of $V_{a} / D$ in the three terminal arrangement. Using the above values and Eq. (1) allows a value of the screening factor, $\eta$, of 0.32 to be calculated for the present configuration. In order to examine whether the value of the screening term is affected by the magnitude of the current, a similar analysis was performed at $1 \mu \mathrm{A}$ current. In the absence of the gated electrode a voltage of $313 \mathrm{~V}$ is required, rising to $460 \mathrm{~V}$, in the presence of the metal electrode. Using the same value of the $\beta$ as before (794) and with the aid of Eq. (1) gives an identical gate screening factor of 0.32. If this screening of the field was ignored, a naive calculation of the local field, assuming an applied voltage of $460 \mathrm{~V}$ and an enhancement factor of 620, would overestimate the local field at $5.27 \mathrm{~V} / \mathrm{nm}$, which is an overestimation by $15 \%$ of the actual local field. This shows that asizable fraction of the applied electric field is screened by the presence of the metallic gate and independent of current. to AIP license or copyright; see http://apl.aip.org/apl/copyright.jsp 

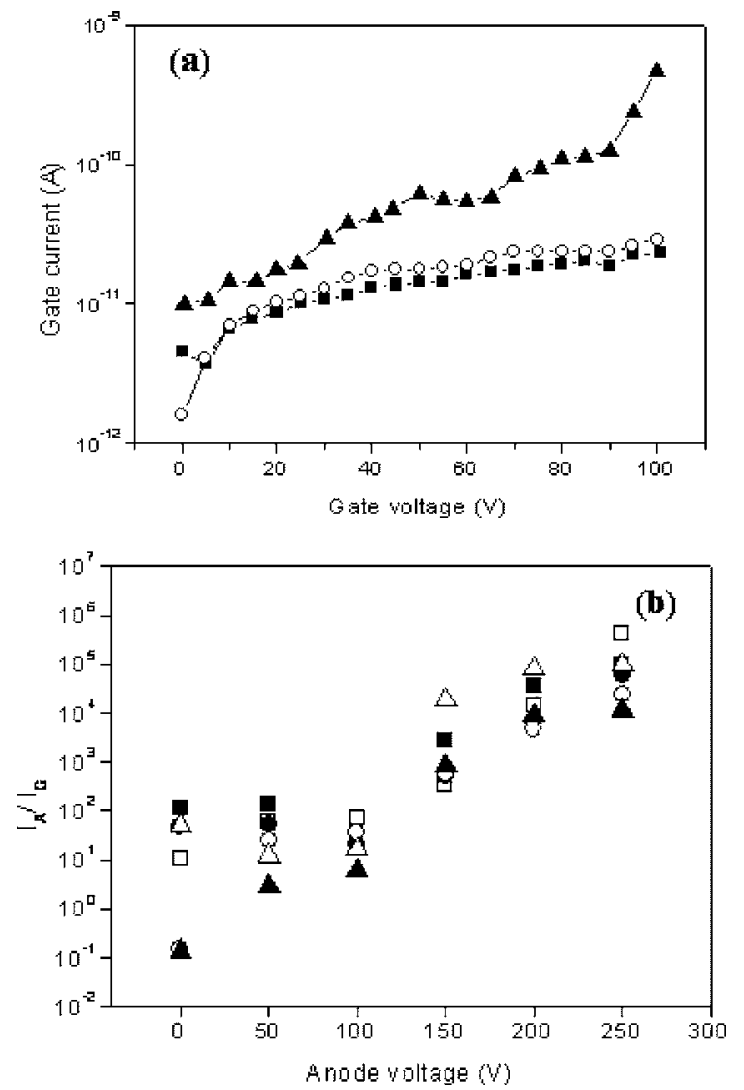

FIG. 3. (a) Variation of gate current against gate voltage for three different

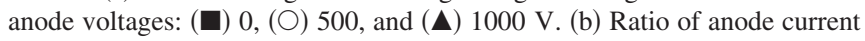
to gate current plotted against anode voltage for gate biases of $0(\mathbb{\square}), 5(\square)$, $10(\bullet), 15(\bigcirc), 20(\boldsymbol{\Delta})$, and $25 \mathrm{~V}(\triangle)$. Up to $V_{A}=100 \mathrm{~V}$ the ratio is between 0.1 and 100; however, at $V_{A}>100 \mathrm{~V}$ and the structure is emitting, this ratio exceeds $10^{6}$.

The decrease in the anode voltage required for $10 \mathrm{nA}$ of current as the gate bias increases [Fig. 2(a)] can be explained by the local field experienced by the nanotube now having a contribution from the gate electrode. In such a situation, electrons being emitted by the nanotube are initially attracted by the gate electrode and are then accelerated towards the anode. This gives rise to the possibility that a fraction of electrons will not reach the anode but will contribute to the gate current. Figure 3(a) shows the variation gate current $\left(I_{G}\right)$ against gate voltage for three values of anode voltage $(0$, 500 , and $1000 \mathrm{~V})$. It is apparent for the two lowest anode voltages investigated that the gate current never exceeds 2 $\times 10^{-11} \mathrm{~A}$, even for gate voltages of $100 \mathrm{~V}$. Only for an anode voltage of $1000 \mathrm{~V}$ is there evidence for an increase of gate current with gate voltage, due to the onset of emission. Such low values of gate current, in contrast to other studies for arrays of emitters, ${ }^{10}$ reflect the intrinsic level of emission, and the in situ manipulation is a way to characterize this intrinsic emission. Figure 3(b) shows the variation of the ratio of anode to gate currents as a function of anode voltage for each gate voltage. At low anode voltages the ratio lies between 0.1 and 100 reflecting the low noise determined level of current present at such low anode voltages, where the onset of emission has not occurred. For anode voltage in excess of $100 \mathrm{~V}$, the ratio steadily increases for all the gate voltages explored, reaching $10^{6}$ for $V_{A}=250 \mathrm{~V}$.

Previous reports on three terminal device structures comprising nanotube, ${ }^{7}$ nanostructured carbon, ${ }^{8}$ and nanofibers 9 (CNFs) as the electron sources also analyzed emission characteristics. Nanotube emitters ${ }^{7}$ when arranged Downloaded 30 Mar 2009 to 131.227 .178 .132 . Redistribution subject individually in a gate well emit $40 \mathrm{nA}$ to the gate electrode at a gate bias of $40 \mathrm{~V}$, which corresponds to a gate transparency, defined as the ratio of emission current collected at the anode to emission current collected at the gate, of $99 \%$. Work on nanostructured carbon ${ }^{8}$ as electron sources utilized a transmission electron microscopy grid as the gate electrode, placed $55 \mu \mathrm{m}$ from the cathode which resulted in high gate turn on voltages of $220 \mathrm{~V}$; however, a gate transparency of only $50 \%$ is calculated at maximum emission of $10 \mu \mathrm{A}$. Guillorn et al. ${ }^{9}$ reported the use of carbon nanofibers as electron sources with a relatively high gate voltage of $85 \mathrm{~V}$ required for an emission from the CNF of $0.3 \mu \mathrm{A}$; however, this corresponds to a high gate transparency of $99 \%$. The results presented in this letter have the following characteristics when considering Figs. 2(a) and 3(a): At a gate voltage of $25 \mathrm{~V}$ the peak anode current of $1 \mu \mathrm{A}$ (at $V_{A}=180 \mathrm{~V}$ ) is observed [Fig. 2(a)]. From Fig. 3(a) the corresponding gate current is $\sim 10^{-11} \mathrm{~A}$. This corresponds to a transparency of $99.99 \%$, which is a more efficient gate structure than seen in previous work. ${ }^{7-9}$ It can also be seen from Fig. 3(a) that when the structure was examined at higher values of $V_{G}$ and $V_{A}(100$ and $1000 \mathrm{~V}$, respectively), an emission to the gate electrode of $5 \times 10^{-10} \mathrm{~A}$ is seen. When considering that the maximum emission current was limited to $1 \mu \mathrm{A}$, this corresponds to a still high gate transparency of $99.95 \%$.

In conclusion, we have demonstrated in situ characterization of three terminal field emission from a single CNT. We have shown that the presence of the gate electrode can screen the applied anode field by about $32 \%$ and that care must be taken in performing Fowler-Nordheim analysis on the $I-V$ characteristic of such gated structures. Our method of manipulation of electrodes can be used to examine the three terminal field emission properties of a range of tip based emitters, provided the tip-to-tip lateral separation is sufficiently large. The ability to manipulate the position of anode and gate electrodes permits measurement and analysis of individual emitters and offers opportunities above those currently available for large area testing of arrays of emitters.

The authors would like to thank the EPSRC Portfolio Partnership for funding this research.

${ }^{1}$ S. Iijima, Nature (London) 56, 354 (1991).

${ }^{2}$ V. Semet, Vu Thien Binh, P. Vincent, D. Guillot, K. B. K. Teo, M. Chhowalla, G. A. J. Amaratunga, W. I. Milne, P. Legagneux, and D. Pribat, Appl. Phys. Lett. 81, 343 (2002).

${ }^{3}$ E. Minoux, O. Groening, K. B. K. Teo, S. H. Dalal, L. Gangloff, J.-P. Schnell, L. Hudanski, I. Y. Y. Bu, P. Vincent, P. Legagneux, G. A. J. Amaratunga, and W. I. Milne, Nano Lett. 5, 2135 (2005).

${ }^{4}$ Y. Saito, K. Hamaguchi, T. Nishina, K. Hata, K. Tohji, A. Kasuya, and Y. Nishina, Jpn. J. Appl. Phys., Part 2 36, L1340 (1997).

${ }^{5}$ R. C. Smith, D. C. Cox, and S. R. P. Silva, Appl. Phys. Lett. 87, 103112 (2005).

${ }^{6}$ L. Nilsson, O. Groening, C. Emmenegger, O. Kuettel, E. Schaller, L. Schlapbach, H. Kind, J.-M. Bonard, and K. Kern, Appl. Phys. Lett. 76, 2071 (2000).

${ }^{7}$ L. Gangloff, E. Minoux, K. B. K. Teo, P. Vincent, V. T. Semet, V. T. Binh, M. H. Yang, I. Y. Y. Bu, R. G. Lacerdera, G. Pirio, J. P. Schnell, D. Pribat, D. G. Hasko, G. A. J. Amaratunga, W. I. Milne, and P. Legagneux, Nano Lett. 4, 1575 (2004).

${ }^{8}$ K. H. Park, H. J. Han, S. Choi, K. M. Lee, S. Lee, and K. H. Koh, J. Vac. Sci. Technol. B 21, 562 (2003).

${ }^{9}$ M. A. Guillorn, A. V. Melechko, V. I. Merkulov, E. D. Ellis, M. K. Simpson, D. H. Lowndes, L. R. Baylor, and G. J. Bordonaro, J. Vac. Sci. Technol. B 19, 2598 (2001).

${ }^{10}$ G. Pirio, P. Legagneux, D. Pribat, K. B. K. Teo, M. Chhowalla, G. A. J. Amaratunga, and W. I. Milne, Nanotechnology 13, 1 (2001).

${ }^{11}$ D. C. Cox, R. D. Forrest, P. R. Smith, V. Stolojan, and S. R. P. Silva, Appl. Phys. Lett. 87, 033102 (2005).

AIP license or copyright; see http://apl.aip.org/apl/copyright.jsp 